\title{
COMMUTATORS OF MAXIMAL FUNCTIONS ON SPACES OF HOMOGENEOUS TYPE AND THEIR WEIGHTED, LOCAL VERSIONS
}

\author{
ZUNWEI FU, ELODIE POZZI AND QINGYAN WU
}

\begin{abstract}
We establish the characterizations of commutators of several versions of maximal functions on spaces of homogeneous type. In addition, with the aid of interpolation theory, we provide weighted version of the commutator theorems by establishing new characterizations of the weighted BMO space. Finally, a concrete example shows the local version of commutators also has an independent interest.
\end{abstract}

\section{INTRODUCTION}

On the Euclidean space, for the Hilbert transform $H$, and other classical singular integral operators, a well-known result due to Coifman, Rochberg and Weiss (cf. [9]) states that a locally integrable function $b$ in $\mathbb{R}^{n}$ is in BMO if and only if the commutator $[H, b] f:=$ $H(b f)-b H(f)$, is bounded in $L^{p}$, for some (and for all) $1<p<\infty$. In [5. Propositions 4 and 6], Bastero, Milman and Ruiz characterized the class of functions for which the commutator with the Hardy-Littlewood maximal function and the maximal sharp function are bounded on $L^{p}$. Later, García-Cuerva et al. [18, Theorem 2.4] proved that the maximal commutator $\mathcal{C}_{b}$ is bounded on $L^{p}\left(\mathbb{R}^{n}\right), 1<p<\infty$, if and only if $b \in \operatorname{BMO}\left(\mathbb{R}^{n}\right)$, where $\mathcal{C}_{b}$ is defined by

$$
\mathcal{C}_{b}(f)(x)=\sup _{Q \ni x} \frac{1}{|Q|} \int_{Q}|b(x)-b(y)||f(y)| d y .
$$

For the endpoint case, Agcayazi, Gogatishvili, Koca and Mustafayev [1, Theorems 1.5, 1.6 and 1.8] proved that the commutator $[\mathcal{M}, b]$ of the Hardy-Littlewood maximal function $\mathcal{M}$ is bounded from $L \log ^{+} L\left(\mathbb{R}^{n}\right)$ into weak $L^{1}\left(\mathbb{R}^{n}\right)$ if and only if $b$ is in $\operatorname{BMO}\left(\mathbb{R}^{n}\right)$. Similar result holds for the maximal commutator $\mathcal{C}_{b}$. Regrading weak type $(1,1)$, we note that in [1, the authors also gave a counterexample to show that $[\mathcal{M}, b]$ fails to be of weak type $(1,1)$.

In this paper, we want to extend the above results to the spaces of homogeneous type. In modern harmonic analysis, it has been a central theme to extend the real variable theory from the Euclidean setting, namely where the underlying space is $\mathbb{R}^{n}$ with the Euclidean metric and Lebesgue measure, to more general settings. To this end, Coifman and Weiss formulated the concept of spaces of homogeneous type, in [10]. There is a large literature devoted to spaces of homogeneous type, see for example [2, 4, 7, 10, 11, 12, 13, 20, 21, 22, 23, 34]. Some non-Euclidean examples of spaces of homogeneous type are given by the CarnotCarathéodory spaces whose theory is developed by Nagel, Stein and others in [37, 38. and related papers; there the quasi-metric is defined in terms of vector fields satisfying the Hörmander condition on an underlying manifold. Very recently, He et. al [25] presented a complete real-variable theory of Hardy spaces on spaces of homogeneous type without additional (geometrical) measure condition in which only doubling condition is required.

Date: July 29, 2019.

2010 Mathematics Subject Classification. Primary 42B35, Secondary 30L99, 42B25.

Key words and phrases. maximal function, space of homogeneous type, BMO space, weight, commutator. 
Recall that $(X, \rho, \mu)$ is a space of homogeneous type in the sense of Coifman and Weiss if $\rho$ is a quasi-metric and $\mu$ is a nonzero measure satisfying the doubling condition. A quasimetric $\rho: X \times X \rightarrow[0, \infty)$ satisfies (i) $\rho(x, y)=\rho(y, x) \geq 0$ for all $x, y \in X$; (ii) $\rho(x, y)=0$ if and only if $x=y$; and (iii) the quasi-triangle inequality: there is a constant $A_{0} \in[1, \infty)$ such that for all $x, y, z \in X$,

$$
\rho(x, y) \leq A_{0}[\rho(x, z)+\rho(z, y)] .
$$

In contrast to a metric, the quasi-metric may not be Hölder regular and quasi-metric balls may not be open; see for example [29, p.5]. A nonzero measure $\mu$ satisfies the doubling condition if there is a constant $C_{\mu}$ such that for all $x \in X$ and all $r>0$,

$$
\mu(B(x, 2 r)) \leq C_{\mu} \mu(B(x, r))<\infty,
$$

where $B(x, r)=\{y \in X: \rho(x, y)<r\}$ with $x \in X$ and $r>0$ are the $\rho$-balls. We point out that the doubling condition (1.2) implies that there exists a positive constant $n$ (the upper dimension of $\mu$ ) such that for all $x \in X, \lambda \geq 1$ and $r>0$,

$$
\mu(B(x, \lambda r)) \leq C_{\mu} \lambda^{n} \mu(B(x, r)) .
$$

Throughout this paper, we assume that $\mu$ satisfies the following conditions: $\mu\left(\left\{x_{0}\right\}\right)=0$ for every $x_{0} \in X$.

We now consider the case $\mu(X)=\infty$ (See also the case $\mu(X)<\infty$ in Section 4). Note that $\operatorname{BMO}(X)$ is defined as the set of all $b \in L_{l o c}^{1}(X)$ such that

$$
\sup _{B \subset X} \frac{1}{\mu(B)} \int_{B}\left|b(y)-m_{B}(b)\right| d \mu(y)<\infty,
$$

where $m_{B}(f)$ is the average of $f$ on $B$, i.e. $m_{B}(f)=\frac{1}{\mu(B)} \int_{B} f(y) d \mu(y)$, and the norm is defined as

$$
\|b\|_{\operatorname{BMO}(X)}:=\sup _{B \subset X} \frac{1}{\mu(B)} \int_{B}\left|b(y)-m_{B}(b)\right| d \mu(y) .
$$

Note that it also has an equivalent norm, defined by

$$
\|b\|_{\mathrm{BMO}(X)}^{\prime}=\sup _{B \subset X} \inf _{c \in \mathbb{R}} \frac{1}{\mu(B)} \int_{B}|b(y)-c| d \mu(y) .
$$

For a locally integrable function $f$ on $X$ and for $1 \leq p<\infty$, the Hardy-Littlewood maximal function $M_{p} f$ of $f$ is defined by

$$
M_{p}(f)(x)=\sup _{B \ni x}\left(\frac{1}{\mu(B)} \int_{B}|f(y)|^{p} d \mu(y)\right)^{\frac{1}{p}},
$$

where the supremum is taken over all balls containing $x$. When $p=1$, we write $M=M_{1}$, which is the classical Hardy-Littlewood maximal operator. For any $f \in L_{l o c}^{1}(X)$ and $x \in X$, let $M^{\sharp} f$ be the sharp maximal function of Fefferman and Stein defined by

$$
M^{\sharp} f(x)=\sup _{B \ni x} \frac{1}{\mu(B)} \int_{B}\left|f(y)-m_{B}(f)\right| d \mu(y) .
$$

For a fixed $\delta \in(0,1)$, any suitable function $f$ and $x \in X$, let

$$
M_{\delta}^{\sharp} f(x):=\left[M^{\sharp}\left(|f|^{\delta}\right)(x)\right]^{\frac{1}{\delta}}, \quad M_{\delta} f(x):=\left[M\left(|f|^{\delta}\right)(x)\right]^{\frac{1}{\delta}} .
$$

Given a measurable function $b$, the commutator of the Hardy-Littlewood maximal operator $M_{p}$ and $b$ is defined by

$$
\left[M_{p}, b\right] f(x):=M_{p}(b f)(x)-b(x) M_{p} f(x)
$$


for all $x \in X .\left[M^{\sharp}, b\right]$ can be defined in the same way. The maximal commutator is defined by

$$
C_{b}(f)(x):=\sup _{B \ni x} \frac{1}{\mu(B)} \int_{B}|b(x)-b(y)||f(y)| d \mu(y)
$$

for all $x \in X$. For any ball $B \subset X$, define

$$
M_{p, B}(f)(x)=\sup _{x \in B_{0} \subseteq B}\left(\frac{1}{\mu\left(B_{0}\right)} \int_{B_{0}}|f(y)|^{p} d \mu(y)\right)^{\frac{1}{p}} .
$$

This paper is organised as follows. In Section 2 we recall some necessary definitions and results on space of homogeneous type. We mainly present the pointwise estimate for $\left[M_{p}, b\right], C_{b}$ and $\left[M^{\sharp}, b\right]$. We also provide a counter example on space of homogeneous type showing that the commutator $[M, b]$ fails to be of weak type $(1,1)$. Section 3 focuses on the weighted version of commutator theorems. In the last section we skim through the local characterizations and give a concrete example on bounded pseudoconvex domain in $\mathbb{C}^{n}$ which shows the local version of commutators also has its independent connotation.

\section{Pointwise estimates}

The poinwise estimates for the characterizations of commutators of maximal functions on spaces of homogeneous type depend on the case of the Euclidean space appeared in [1]. However, this is the basis for later weighted and local estimations. Therefore, we will still give a complete proof with the aid of proof based on [1] to keep the integrity of the paper.

Theorem 2.1. Let $b$ be a real valued, locally integrable function in X. The following assertions are equivalent:

(i) $b \in \operatorname{BMO}(X)$ and $b^{-} \in L^{\infty}(X)$;

(ii) The commutator $\left[M_{p}, b\right]$ is bounded on $L^{q}(X)$, for all $q, p<q<\infty$;

(iii) The commutator $\left[M_{p}, b\right]$ is bounded on $L^{q}(X)$, for some $q, p<q<\infty$;

(iv) For all $q \in[1, \infty)$ we have

$$
\sup _{B} \frac{1}{\mu(B)} \int_{B}\left|b(x)-M_{p, B}(b)(x)\right|^{q} d \mu(x)<\infty .
$$

(v) There exists $q \in[1, \infty)$ such that

$$
\sup _{B} \frac{1}{\mu(B)} \int_{B}\left|b(x)-M_{p, B}(b)(x)\right|^{q} d \mu(x)<\infty .
$$

Theorem 2.2. Let $b$ be a real valued, locally integrable function in $X$. The following assertions are equivalent:

(i) $b \in \operatorname{BMO}(X)$ and $b^{-} \in L^{\infty}(X)$;

(ii) The commutator $\left[M^{\sharp}, b\right]$ is bounded on $L^{q}(X, \mu)$, for all $q, 1<q<\infty$;

(iii) The commutator $\left[M^{\sharp}, b\right]$ is bounded on $L^{q}(X, \mu)$, for some $q, 1<q<\infty$;

(iv) For all $q \in[1, \infty)$, we have

$$
\sup _{B} \frac{1}{\mu(B)} \int_{B}\left|b(x)-2 M^{\sharp}\left(b \chi_{B}\right)(x)\right|^{q} d \mu(x)<\infty ;
$$

(v) There exists $q \in[1, \infty)$ such that

$$
\sup _{B} \frac{1}{\mu(B)} \int_{B}\left|b(x)-2 M^{\sharp}\left(b \chi_{B}\right)(x)\right|^{q} d \mu(x)<\infty .
$$

Theorem 2.3. Let $b \in \operatorname{BMO}(X)$ such that $b^{-} \in L^{\infty}(X)$. Then there exists a positive constant $C$ such that for all $f \in L\left(1+\log ^{+} L\right)(X)$ and $\lambda>0$,

$\mu(\{x \in X:|[M, b] f(x)|>\lambda\})$ 
$\leq C\left(\left\|b^{+}\right\|_{\mathrm{BMO}(X)}+\left\|b^{-}\right\|_{L^{\infty}}\right)\left(1+\log ^{+}\left(\left\|b^{+}\right\|_{\mathrm{BMO}(X)}\right)\right) \int_{X} \frac{|f(x)|}{\lambda}\left(1+\log ^{+}\left(\frac{|f(x)|}{\lambda}\right)\right) d \mu(x)$.

Theorem 2.4. Let $b \in L_{l o c}^{1}(X)$ and $1<p<\infty$. Then the maximal commutator $C_{b}$ is bounded on $L^{p}(X)$ if and only if $b \in \mathrm{BMO}(\mathrm{X})$.

Theorem 2.5. Let $b \in L_{\text {loc }}^{1}(X)$. Then $b \in \operatorname{BMO}(X)$ if and only if there exists a positive constant $C$ such that for each $\lambda>0$ and all $f \in L\left(1+\log ^{+} L\right)(X)$,

$$
\mu\left(\left\{x \in X: C_{b}(f)(x)>\lambda\right\}\right) \leq C \int_{X} \frac{|f(x)|}{\lambda}\left(1+\log ^{+}\left(\frac{|f(x)|}{\lambda}\right)\right) d \mu(x) .
$$

We mention here the sufficiency of $b \in \operatorname{BMO}(X)$ for the boundedness of $C_{b}$ in Theorem 2.4 and Theorem 2.5 has been studied by Hu, Lin and Yang (see [27]) in a different way. We will give the proof of the converse part.

For any locally integrable function $f$, let $f_{\mu}^{*}$ be the non-increasing rearrangement of $f$ (see for example [33]), namely,

$$
f_{\mu}^{*}(t)=\inf \{s>0: \mu(\{x \in X:|f(x)|>s\})<t\}, \quad 0<t<\infty .
$$

The following John-Nirenberg inequalities on spaces of homogeneous type come from 31, Propositions 6, 7].

Lemma 2.6. If $f \in \operatorname{BMO}(X)$, then there exist positive constants $C_{1}$ and $C_{2}$ such that for every ball $B \subset X$ and every $\alpha>0$, we have

$$
\mu\left(\left\{x \in B:\left|f(x)-m_{B}(f)\right|>\alpha\right\}\right) \leq C_{1} \mu(B) \exp \left\{-\frac{C_{2}}{\|f\|_{\mathrm{BMO}(X)}} \alpha\right\} .
$$

Lemma 2.7. If $f \in \mathrm{BMO}(X)$, then there exist positive constants $C_{3}$ and $C_{4}$ such that for every ball $B \subset X$,

$$
\int_{B} \exp \left\{C_{3}\left|f(x)-m_{B}(f)\right|\right\} d \mu(x)<C_{4} \mu(B) .
$$

On spaces of homogeneous type, we also have the following equivalent BMO norm, see for example [24, Theorem 5.5].

Lemma 2.8. Let $0<p<\infty$, and $f$ be a measurable function on $X$. Then $f$ is in $L_{\text {loc }}^{1}(X)$ and satisfies

$$
\sup _{B}\left(\frac{1}{\mu(B)} \int_{B}\left|f(x)-m_{B}(f)\right|^{p} d \mu(x)\right)^{\frac{1}{p}}<\infty
$$

if and only if $f$ is in $\mathrm{BMO}(X)$. In such a case, we have

$$
\|f\|_{\mathrm{BMO}(X)} \approx \sup _{B}\left(\frac{1}{\mu(B)} \int_{B}\left|f(x)-m_{B}(f)\right|^{p} d \mu(x)\right)^{\frac{1}{p}} .
$$

A generalized Hölder's inequality will be used in our argument. For any measurable set $E$ with $\mu(E)<\infty$, for any suitable function $f$, the norm $\|f\|_{L \log L, E}$ is defined by

$$
\|f\|_{L \log L, E}=\inf \left\{\lambda>0: \frac{1}{\mu(E)} \int_{E} \frac{|f(y)|}{\lambda} \log \left(2+\frac{|f(y)|}{\lambda}\right) d \mu(y) \leq 1\right\} .
$$

The maximal operator $M_{L \log L}$ is defined by

$$
M_{L \log L} f(x)=\sup _{B \ni x}\|f\|_{L \log L, B} .
$$

The norm $\|f\|_{\exp L, E}$ is defined by

$$
\|f\|_{\exp L, E}=\inf \left\{\lambda>0: \frac{1}{\mu(E)} \int_{E} \exp \left(\frac{|f(x)|}{\lambda}\right) d \mu(x) \leq 2\right\} .
$$


Then the following generalized Hölder's inequality:

$$
\frac{1}{\mu(E)} \int_{E}|f(x) g(x)| d \mu(x) \leq C\|f\|_{L \log L, E}\|g\|_{\exp L, E}
$$

holds for any suitable functions $f$ and $g$ (see for example [41]). From [39, p. 90], we can see

$$
M^{2} f(x) \approx M_{L \log L} f(x),
$$

where $M^{2}=M \circ M$.

Lemma 2.9 ([8]). There is a positive constant $C$ such that for any bounded function $f$ with bounded support and for all $\lambda>0$,

$$
\mu\left(\left\{y \in X: M_{L \log L} f(x)>\lambda\right\}\right) \leq C \int_{X} \frac{|f(y)|}{\lambda}\left(1+\log ^{+}\left(\frac{|f(y)|}{\lambda}\right)\right) d \mu(y) .
$$

We need the following relation between operators $C_{b}$ and $[M, b]$. The proofs are similar to those of Lemma 3.1 and Lemma 3.2 in [1], respectively.

Lemma 2.10. Let $b$ be any non-negative locally integrable function. Then

$$
|[M, b] f(x)| \leq C_{b}(f)(x)
$$

for all $f \in L_{l o c}^{1}(X)$.

Lemma 2.11. Let $b$ be any locally integrable function on $X$. Then

$$
|[M, b] f(x)| \leq C_{b}(f)(x)+2 b^{-}(x) M f(x)
$$

holds for all $f \in L_{\text {loc }}^{1}(X)$.

Proposition 2.12. Let $b \in \operatorname{BMO}(X)$ and let $0<\delta<1$. Then there exists a positive constant $C=C(\delta)$ such that

$$
M_{\delta}\left(C_{b}(f)\right)(x) \leq C\|b\|_{\mathrm{BMO}(X)} M^{2} f(x), \quad x \in X,
$$

for all $f \in L_{l o c}^{1}(X)$.

Proof of Proposition 2.12. Let $x \in X$ and fix a ball $B$ containing $x$. Let $f=f_{1}+f_{2}$, where $f_{1}=f \chi_{3 B}$. For any $y \in X$, we have

$$
\begin{aligned}
C_{b}(f)(y) & =M((b-b(y)) f)(y)=M\left(\left(b-m_{3 B}(b)+m_{3 B}(b)-b(y)\right) f\right)(y) \\
& \leq M\left(\left(b-m_{3 B}(b)\right) f\right)(y)+\left|m_{3 B}(b)-b(y)\right| M f(y) \\
& \leq M\left(\left(b-m_{3 B}(b)\right) f_{1}\right)(y)+M\left(\left(b-m_{3 B}(b)\right) f_{2}\right)(y)+\left|m_{3 B}(b)-b(y)\right| M f(y) .
\end{aligned}
$$

Therefore,

$$
\begin{aligned}
\left(\frac{1}{\mu(B)} \int_{B}\left(C_{b}(f)(y)\right)^{\delta} d \mu(y)\right)^{\frac{1}{\delta}} \leq & \left(\frac{1}{\mu(B)} \int_{B}\left|M\left(\left(b-m_{3 B}(b)\right) f_{1}\right)(y)\right|^{\delta} d \mu(y)\right)^{\frac{1}{\delta}} \\
& +\left(\frac{1}{\mu(B)} \int_{B}\left|M\left(\left(b-m_{3 B}(b)\right) f_{2}\right)(y)\right|^{\delta} d \mu(y)\right)^{\frac{1}{\delta}} \\
& +\left(\frac{1}{\mu(B)} \int_{B}\left|m_{3 B}(b)-b(y)\right|^{\delta}(M f(y))^{\delta} d \mu(y)\right)^{\frac{1}{\delta}} \\
= & I+I I+I I I .
\end{aligned}
$$

We first estimate $I$. Recall that $M$ is weak-type $(1,1)$ (c.f. [19, P. 299]). We have

$$
\int_{B}\left|M\left(\left(b-m_{3 B}(b)\right) f_{1}\right)(y)\right|^{\delta} d \mu(y) \leq \int_{0}^{\mu(B)}\left[\left(M\left(\left(b-m_{3 B}(b)\right) f_{1}\right)\right)^{*}(t)\right]^{\delta} d t
$$




$$
\begin{aligned}
& \leq\left[\sup _{0<t<\mu(B)} t\left(M\left(\left(b-m_{3 B}(b)\right) f_{1}\right)\right)^{*}(t)\right]^{\delta} \int_{0}^{\mu(B)} t^{-\delta} d t \\
& \leq C\left\|\left(b-m_{3 B}(b)\right) f_{1}\right\|_{L^{1}(X)}^{\delta} \mu(B)^{-\delta+1} \\
& \leq C\left\|\left(b-m_{3 B}(b)\right) f\right\|_{L^{1}(3 B)}^{\delta} \mu(B)^{-\delta+1} .
\end{aligned}
$$

Thus

$$
I \leq C \frac{1}{\mu(B)} \int_{3 B}\left|b(y)-m_{3 B}(b)\right||f(y)| d \mu(y) .
$$

Then by (2.1) and the John-Nirenberg inequality, we have

$$
\begin{aligned}
I & \leq C\left\|b-m_{3 B}(b)\right\|_{\exp L, 3 B}\|f\|_{L \log L, 3 B} \\
& \leq C\|b\|_{\mathrm{BMO}(X)} M_{L \log L} f(x) .
\end{aligned}
$$

For $I I$, since for any two points $x, y \in B$, we have

$$
M\left(\left(b-m_{3 B}(b)\right) f\right)(y) \leq C M\left(\left(b-m_{3 B}(b)\right) f\right)(x)
$$

with $C$ an absolute constant (see for example [17, p. 160]). Then by (2.1), we can get

$$
\begin{aligned}
I I & \leq C M\left(\left(b-m_{3 B}(b)\right) f\right)(x) \\
& =\sup _{B \ni x} \frac{1}{\mu(B)} \int_{B}\left|b(y)-m_{3 B}(b) \| f(y)\right| d \mu(y) \\
& \leq C \sup _{B \ni x}\left\|b-m_{3 B}(b)\right\|_{\exp L, 3 B}\|f\|_{L \log L, 3 B} \\
& \leq C\|b\|_{\mathrm{BMO}(X)} M_{L \log L} f(x) .
\end{aligned}
$$

For $I I I$, by Hölder's inequality, Lemma 2.8, we have

$$
\begin{aligned}
I I I & =\left(\frac{1}{\mu(B)} \int_{B}\left|m_{3 B}(b)-b(y)\right|^{\delta}(M f(y))^{\delta} d \mu(y)\right)^{\frac{1}{\delta}} \\
& \leq\left(\frac{1}{\mu(B)} \int_{B}\left|m_{3 B}(b)-b(y)\right|^{\frac{\delta}{1-\delta}} d \mu(y)\right)^{\frac{1-\delta}{\delta}}\left(\frac{1}{\mu(B)} \int_{B}(M f(y)) d \mu(y)\right) \\
& \leq C\|b\|_{\mathrm{BMO}(X)} M^{2} f(x) .
\end{aligned}
$$

Therefore, by (2.2), we have

$$
\begin{aligned}
\left(\frac{1}{\mu(B)} \int_{B}\left(C_{b}(f)(y)\right)^{\delta} d \mu(y)\right)^{\frac{1}{\delta}} & \leq C\|b\|_{\mathrm{BMO}(X)}\left(M_{L \log L} f(x)+M^{2} f(x)\right) \\
& \leq C\|b\|_{\mathrm{BMO}(X)} M^{2} f(x) .
\end{aligned}
$$

This finishes the proof of Proposition 2.12,

By the Lebesgue differentiation theorem, we can get the following corollary.

Corollary 2.13. Let $b \in \operatorname{BMO}(X)$. Then there exists a positive constant $C$ such that for all $f \in L_{l o c}^{1}(X)$,

$$
C_{b}(f)(x) \leq C\|b\|_{\mathrm{BMO}(X)} M^{2} f(x), \quad x \in X .
$$

Corollary 2.14. Let $b \in \operatorname{BMO}(X)$ such that $b^{-} \in L^{\infty}(X)$. Then there exists a positive constant $C$ such that for all $f \in L_{\text {loc }}^{1}(X)$,

$$
|[M, b] f(x)| \leq C\left(\left\|b^{+}\right\|_{\mathrm{BMO}(X)}+\left\|b^{-}\right\|_{L^{\infty}}\right) M^{2} f(x) .
$$


Proof. By Lemma 2.11, Corollary 2.13 and the fact that $f \leq M f$, we have

$$
\begin{aligned}
|[M, b] f(x)| & \leq C_{b}(f)(x)+2 b^{-}(x) M f(x) \\
& \leq C\left(\|b\|_{\mathrm{BMO}(X)} M^{2} f(x)+b^{-}(x) M f(x)\right) \\
& \leq C\left[\left(\left\|b^{+}\right\|_{\mathrm{BMO}(X)}+\left\|b^{-}\right\|_{\mathrm{BMO}(X)}\right) M^{2} f(x)+\left\|b^{-}\right\|_{L^{\infty}(X)} M(M f)(x)\right] \\
& \leq C\left(\left\|b^{+}\right\|_{\mathrm{BMO}(X)}+\left\|b^{-}\right\|_{L^{\infty}}\right) M^{2} f(x) .
\end{aligned}
$$

This finishes the proof.

According to [35, Theorem 4.4], we have the following boundedness result for quasilinear operators $T$, which satisfy

(a) $T f \geq 0$, for $f \in D(T)$;

(b) $T(\alpha f)=|\alpha| T f$, for $\alpha \in \mathbb{R}$ and $f \in D(T)$;

(c) $|T f-T g| \leq T(f-g)$, for $f, g \in D(T)$,

where $D(T)$ is a suitable class of locally integrable functions.

Lemma 2.15. Let $b \in \mathrm{BMO}(X)$ be a nonnegative function. Suppose that $T$ is a quasilinear operator satisfying (a)-(c) and is bounded on $L^{q}(X)$, for some $1 \leq q<\infty$. Then $[T, b]$ is bounded on $L^{q}(X)$.

Since $|[T, b] f-[T,|b|] f| \leq 2\left(b^{-} T(f)+T\left(b^{-} f\right)\right)$, for general BMO function $b$, the following result holds (see also [5, Proposition 3])

Proposition 2.16. Let $b \in \mathrm{BMO}(\mathrm{X})$ with $b^{-} \in L^{\infty}(X)$. Suppose that $T$ is a quasilinear operator satisfying (a)-(c) and is bounded on $L^{q}(X)$, for some $1 \leq q<\infty$. Then $[T, b]$ is bounded on $L^{q}(X)$.

Proof of Theorem 2.1. It is clear that (ii) implies (iii), (iv) implies (v). For (i) $\Rightarrow\left(\right.$ ii), since $M_{p}$ satisfies (a)-(c) and is bounded on $L^{q}(X)$ for $p<q<\infty$, the result follows from Proposition 2.16. For (iii) $\Rightarrow\left(\mathrm{v}\right.$ ), by assumption, $\left[M_{p}, b\right]$ is bounded on $L^{q}(X)$ for some $p<q<\infty$, then for any fixed ball $B \subset X$, we have

$$
\left(\int_{B}\left|M_{p}(b f)-b M_{p}(f)\right|^{q} d \mu\right)^{\frac{1}{q}} \leq\left\|\left[M_{p}, b\right] f\right\|_{L^{q}(X)} \leq C\|f\|_{L^{q}(X)} .
$$

Now we choose $f=\chi_{B} \in L^{q}(X)$, then $M_{p}\left(\chi_{B}\right)=\chi_{B}$ and $M_{p}\left(b \chi_{B}\right)(x)=M_{p, B}(b)(x)$ for all $x \in B$, thus by (2.4), we have

$$
\left(\int_{B}\left|b-M_{p, B}(b)\right|^{q} d \mu\right)^{\frac{1}{q}} \leq C \mu(B)^{\frac{1}{q}},
$$

which implies (v).

For $(\mathrm{v}) \Rightarrow(\mathrm{i})$, let $B$ be a fixed ball. By Hölder's inequality, we have

$$
\frac{1}{\mu(B)} \int_{B}\left|b-M_{p, B}(b)\right| d \mu \leq\left(\frac{1}{\mu(B)} \int_{B}\left|b-M_{p, B}(b)\right|^{q} d \mu\right)^{\frac{1}{q}} \leq C .
$$

Let $E_{1}=\left\{x \in B: b(x) \leq m_{B}(b)\right\}$ and $E_{2}=\left\{x \in B: b(x)>m_{B}(b)\right\}$. It is clear that

$$
\int_{E_{1}}\left|b-m_{B}(b)\right| d \mu=\int_{E_{2}}\left|b-m_{B}(b)\right| d \mu .
$$

Since for $x \in E_{1}, b(x) \leq m_{B}(b) \leq M_{p, B}(b)(x)$, we have

$$
\begin{aligned}
\frac{1}{\mu(B)} \int_{B}\left|b-m_{B}(b)\right| d \mu & =\frac{2}{\mu(B)} \int_{E_{1}}\left|b-m_{B}(b)\right| d \mu \leq \frac{2}{\mu(B)} \int_{E_{1}}\left|b-M_{p, B}(b)\right| d \mu \\
& \leq \frac{2}{\mu(B)} \int_{B}\left|b-M_{p, B}(b)\right| d \mu \leq C .
\end{aligned}
$$


Therefore, $b \in \operatorname{BMO}(X)$. Next, we will show that $b^{-} \in L^{\infty}(X)$. Observe that $M_{p, B}(b) \geq|b|$ in $B$, therefore, in $B$, we have

$$
0 \leq b^{-} \leq M_{p, B}(b)-b^{+}+b^{-}=M_{p, B}(b)-b .
$$

Together with the assumption of (v), we can see that there exists a constant $C$ such that for any ball $B$, we have

$$
m_{B}\left(b^{-}\right) \leq C .
$$

Then the boundedness of $b^{-}$follows from Lebesgue's differentiation theorem. The implication of (ii) $\Rightarrow$ (iv) is similar to (iii) $\Rightarrow(v)$. This ends the proof of Theorem 2.1.

Proof of Theorem 2.2. Obviously, (ii) $\Rightarrow$ (iii), and (iv) $\Rightarrow(v)$. For (i) $\Rightarrow\left(\right.$ ii), since $M^{\sharp} f \leq 2 M f$ and $M^{\sharp}$ satisfies (a)-(c), the result follows from Proposition 2.16, For (iii) $\Rightarrow(v)$. Let $B$ be a fixed ball and $B_{1}$ be any other ball. Then

$$
\begin{aligned}
& \frac{1}{\mu\left(B_{1}\right)} \int_{B_{1}}\left|\chi_{B}(x)-m_{B_{1}}\left(\chi_{B}\right)\right| d \mu(x) \\
& =\frac{1}{\mu\left(B_{1}\right)} \int_{B_{1}}\left|\frac{1}{\mu\left(B_{1}\right)} \int_{B_{1}}\left(\chi_{B}(x)-\chi_{B}(y)\right) d \mu(y)\right| d \mu(x) \\
& =\frac{1}{\mu\left(B_{1}\right)^{2}}\left(\int_{B_{1} \backslash B} \int_{B_{1} \cap B} d \mu(y) d \mu(x)+\int_{B_{1} \cap B} \int_{B_{1} \backslash B} d \mu(y) d \mu(x)\right) \\
& =\frac{2 \mu\left(B_{1} \backslash B\right) \mu\left(B_{1} \cap B\right)}{\mu\left(B_{1}\right)^{2}} \leq \frac{1}{2} .
\end{aligned}
$$

Therefore,

$$
M^{\sharp}\left(\chi_{B}\right)(x)=\sup _{x \in B_{1}} \frac{1}{\mu\left(B_{1}\right)} \int_{B_{1}}\left|\chi_{B}-m_{B_{1}}\left(\chi_{B}\right)\right| d \mu \leq \frac{1}{2}
$$

On the other hand, given $x \in B$, there always exists a ball $B_{1} \supset B$ such that $\mu\left(B_{1}\right)=$ $2 \mu(B)$. Therefore,

$$
M^{\sharp}\left(\chi_{B}\right)(x)=\frac{1}{2}, \quad x \in B .
$$

By the assumption of (iii), we can see that

$$
\left(\int_{B}\left|M^{\sharp}(b f)-b M^{\sharp}(f)\right|^{q} d \mu\right)^{\frac{1}{q}} \leq\left\|\left[M^{\sharp}, b\right] f\right\|_{L^{q}(X)} \leq\left\|\left[M^{\sharp}, b\right]\right\|_{L^{q}(X) \rightarrow L^{q}(X)}\|f\|_{L^{q}(X)} .
$$

Now we choose $f=\chi_{B} \in L^{q}(X)$, then $M^{\sharp}\left(\chi_{B}\right)(x)=\frac{1}{2}$ for $x \in B$. Therefore,

$$
\left(\int_{B}\left|\frac{1}{2} b(x)-M^{\sharp}\left(b \chi_{B}\right)(x)\right|^{q} d \mu(x)\right)^{\frac{1}{q}}<\left\|\left[M^{\sharp}, b\right]\right\|_{L^{q}(X) \rightarrow L^{q}(X)} \mu(B)^{\frac{1}{q}},
$$

which implies (iv). For (v) $\Rightarrow(\mathrm{i})$, we first prove that

$$
\left|m_{B}(b)\right| \leq 2 M^{\sharp}\left(b \chi_{B}\right)(x), \quad x \in B .
$$

In fact, let $x \in B$ and take $B_{1} \supset B$ satisfying $\mu\left(B_{1}\right)=2 \mu(B)$. Then

$$
\begin{array}{r}
M^{\sharp}\left(b \chi_{B}\right)(x) \geq \frac{1}{\mu\left(B_{1}\right)} \int_{B_{1}}\left|b(y) \chi_{B}(y)-m_{B_{1}}\left(b \chi_{B}\right)\right| d \mu(y) \\
=\frac{1}{\mu\left(B_{1}\right)}\left[\int_{B}\left|b(y) \chi_{B}(y)-m_{B_{1}}\left(b \chi_{B}\right)\right| d \mu(y)\right. \\
\left.\quad+\int_{B_{1} \backslash B}\left|b(y) \chi_{B}(y)-m_{B_{1}}\left(b \chi_{B}\right)\right| d \mu(y)\right]
\end{array}
$$




$$
=\frac{1}{2 \mu(B)}\left[\int_{B}\left|b(y)-m_{B_{1}}\left(b \chi_{B}\right)\right| d \mu(y)+\left|m_{B_{1}}\left(b \chi_{B}\right)\right| \mu\left(B_{1} \backslash B\right)\right] .
$$

By definition,

$$
\begin{aligned}
m_{B_{1}}\left(b \chi_{B}\right) & =\frac{1}{\mu\left(B_{1}\right)} \int_{B_{1}} b(y) \chi_{B}(y) d \mu(y) \\
& =\frac{1}{2 \mu(B)}\left[\int_{B_{1} \backslash B} b(y) \chi_{B}(y) d \mu(y)+\int_{B} b(y) \chi_{B}(y) d \mu(y)\right]=\frac{1}{2} m_{B}(b) .
\end{aligned}
$$

Therefore, we have

$$
\begin{aligned}
M^{\sharp}\left(b \chi_{B}\right)(x) & \geq \frac{1}{2 \mu(B)}\left[\int_{B}\left|b(y)-\frac{1}{2} m_{B}(b)\right| d \mu(y)+\frac{1}{2}\left|m_{B}(b)\right| \mu\left(B_{1} \backslash B\right)\right] \\
& =\frac{1}{2 \mu(B)} \int_{B}\left|b(y)-\frac{1}{2} m_{B}(b)\right| d \mu(y)+\frac{1}{4}\left|m_{B}(b)\right| .
\end{aligned}
$$

On the other hand,

$$
\left|m_{B}(b)\right| \leq \frac{1}{\mu(B)} \int_{B}\left|b(y)-\frac{1}{2} m_{B}(b)\right| d \mu(y)+\frac{1}{2}\left|m_{B}(b)\right| .
$$

Thus

$$
\left|m_{B}(b)\right| \leq 2 M^{\sharp}\left(b \chi_{B}\right)(x) .
$$

Now we show that $b \in \operatorname{BMO}(X)$. Indeed, let $E=\left\{x \in B: b(x) \leq m_{B}(b)\right\}$. Then

$$
\begin{aligned}
\frac{1}{\mu(B)} \int_{B}\left|b(x)-m_{B}(b)\right| d \mu(x) & =\frac{2}{\mu(B)} \int_{E}\left(b(x)-m_{B}(b)\right) d \mu(x) \\
& \leq \frac{2}{\mu(B)} \int_{E}\left(2 M^{\sharp}\left(b \chi_{B}\right)(x)-b(x)\right) d \mu(x) \\
& =\frac{2}{\mu(B)} \int_{E}\left|2 M^{\sharp}\left(b \chi_{B}\right)(x)-b(x)\right| d \mu(x) \\
& \leq \frac{2}{\mu(B)} \int_{B}\left|2 M^{\sharp}\left(b \chi_{B}\right)(x)-b(x)\right| d \mu(x) \leq C .
\end{aligned}
$$

Next we prove that $b^{-} \in L^{\infty}(X)$. Using the inequality

$$
2 M^{\sharp}\left(b \chi_{B}\right)(x)-b(x) \geq\left|m_{B}(b)\right|-b^{+}(x)+b^{-}(x), \quad x \in B,
$$

we arrive at

$$
\begin{aligned}
C & \geq \frac{1}{\mu(B)} \int_{B}\left|2 M^{\sharp}\left(b \chi_{B}\right)(x)-b(x)\right| d \mu(x) \\
& \geq \frac{1}{\mu(B)} \int_{B}\left(2 M^{\sharp}\left(b \chi_{B}\right)(x)-b(x)\right) d \mu(x) \\
& \geq \frac{1}{\mu(B)} \int_{B}\left(\left|m_{B}(b)\right|-b^{+}(x)+b^{-}(x)\right) d \mu(x) \\
& =\left|m_{B}(b)\right|-\frac{1}{\mu(B)} \int_{B} b^{+}(x) d \mu(x)+\frac{1}{\mu(B)} \int_{B} b^{-}(x) d \mu(x) .
\end{aligned}
$$

Letting $\mu(B) \rightarrow 0$ with $x \in B$, by Lebesgue differentiation theorem, we have

$$
c \geq|b(x)|-b^{+}(x)+b^{-}(x)=2 b^{-}(x) .
$$

The implication of (ii) $\Rightarrow$ (iv) is similar to (iii) $\Rightarrow(\mathrm{v})$. This finishes the proof of Theorem 2.2 , 
Proof of Theorem 2.4. Sufficient condition: Assume that $b \in \operatorname{BMO}(X)$, by Corollary 2.13 and the fact that $M$ is bounded on $L^{p}(X)$, we can see that, for every $f \in L^{p}(X), 1<p<\infty$,

$$
\left\|C_{b}(f)\right\|_{L^{p}(X)} \leq C\|b\|_{\mathrm{BMO}(X)}\left\|M^{2} f\right\|_{L^{p}(X)} \leq C\|b\|_{\mathrm{BMO}(X)}\|f\|_{L^{p}(X)} .
$$

Necessary condition: Assume that $C_{b}$ is bounded on $L^{p}(X)$, next we will show that $b \in \operatorname{BMO}(X)$. By Hölder's inequality, we have

$$
\begin{aligned}
\inf _{c \in \mathbb{R}} \int_{B}|b(x)-c| d \mu(x) & \leq \inf _{y \in B} \int_{B}|b(x)-b(y)| d \mu(x) \\
& \leq \frac{1}{\mu(B)} \int_{B} \int_{B}|b(x)-b(y)| d \mu(x) d \mu(y) \\
& \leq \mu(B)^{\frac{1}{p}}\left[\int_{B}\left(\frac{1}{\mu(B)} \int_{B}|b(x)-b(y)| d \mu(y)\right)^{p} d \mu(x)\right]^{\frac{1}{p}} .
\end{aligned}
$$

Since for any $x \in B$,

$$
\frac{1}{\mu(B)} \int_{B}|b(x)-b(y)| d \mu(y) \leq C_{b}\left(\chi_{B}\right)(x)
$$

We can obtain that

$$
\begin{aligned}
\inf _{c \in \mathbb{R}} \int_{B}|b(x)-c| d \mu(x) & \leq \mu(B)^{\frac{1}{p^{\prime}}}\left(\int_{B}\left|C_{b}\left(\chi_{B}\right)(x)\right|^{p} d \mu(x)\right)^{\frac{1}{p}} \\
& \leq \mu(B)\left\|C_{b}\right\|_{L^{p}(X) \rightarrow L^{p}(X) .}
\end{aligned}
$$

Therefore, $b \in \operatorname{BMO}(X)$, and

$$
\|b\|_{\mathrm{BMO}(X)} \leq C\left\|C_{b}\right\|_{L^{p}(X) \rightarrow L^{p}(X)} .
$$

This completes the proof of the theorem.

Proof of Theorem 2.5. Sufficient condition: Let $B_{0}$ be any fixed ball and $f=\chi_{B_{0}}$. For any $\lambda>0$, we have

$$
\begin{aligned}
\mu\left(\left\{x \in X: C_{b}(f)(x)>\lambda\right\}\right) & =\mu\left(\left\{x \in X: \sup _{B \ni x} \frac{1}{\mu(B)} \int_{B \cap B_{0}}|b(x)-b(y)| d \mu(y)>\lambda\right\}\right) \\
& \geq \mu\left(\left\{x \in B_{0}: \sup _{B \ni x} \frac{1}{\mu(B)} \int_{B \cap B_{0}}|b(x)-b(y)| d \mu(y)>\lambda\right\}\right) \\
& \geq \mu\left(\left\{x \in B_{0}: \frac{1}{\mu\left(B_{0}\right)} \int_{B_{0}}|b(x)-b(y)| d \mu(y)>\lambda\right\}\right) \\
& \geq \mu\left(\left\{x \in B_{0}:\left|b(x)-m_{B_{0}}(b)\right|>\lambda\right\}\right) .
\end{aligned}
$$

By assumption, we have

$$
\begin{aligned}
\mu\left(\left\{x \in B_{0}:\left|b(x)-m_{B_{0}}(b)\right|>\lambda\right\}\right) & \leq C \int_{X} \frac{|f(x)|}{\lambda}\left(1+\log ^{+}\left(\frac{|f(x)|}{\lambda}\right)\right) d \mu(x) \\
& =C \frac{1}{\lambda}\left(1+\log ^{+} \frac{1}{\lambda}\right) \mu\left(B_{0}\right) .
\end{aligned}
$$

Then by Lemma 2.8, for $0<p<1$, we have

$$
\begin{aligned}
\int_{B_{0}}\left|b(x)-m_{B_{0}}(b)\right|^{p} d \mu(x)= & p \int_{0}^{\infty} \lambda^{p-1} \mu\left(\left\{x \in B_{0}:\left|b(x)-m_{B_{0}}(b)\right|>\lambda\right\}\right) d \lambda \\
= & p \int_{0}^{1} \lambda^{p-1} \mu\left(\left\{x \in B_{0}:\left|b(x)-m_{B_{0}}(b)\right|>\lambda\right\}\right) d \lambda \\
& +p \int_{1}^{\infty} \lambda^{p-1} \mu\left(\left\{x \in B_{0}:\left|b(x)-m_{B_{0}}(b)\right|>\lambda\right\}\right) d \lambda
\end{aligned}
$$




$$
\begin{aligned}
& \leq p \mu\left(B_{0}\right) \int_{0}^{1} \lambda^{p-1} d \lambda+C p \mu\left(B_{0}\right) \int_{1}^{\infty} \lambda^{p-1} \frac{1}{\lambda}\left(1+\log ^{+} \frac{1}{\lambda}\right) d \lambda \\
& =\mu\left(B_{0}\right)+C p \mu\left(B_{0}\right) \int_{1}^{\infty} \lambda^{p-2} d \lambda \\
& =\left(1+C \frac{p}{1-p}\right) \mu\left(B_{0}\right) .
\end{aligned}
$$

Therefore,

$$
\left(\frac{1}{\mu\left(B_{0}\right)} \int_{B_{0}}\left|b(x)-m_{B_{0}}(b)\right|^{p} d \mu(x)\right)^{\frac{1}{p}} \leq\left(1+C \frac{p}{1-p}\right)^{\frac{1}{p}} .
$$

Then it follows from Lemma 2.8 that $b \in \operatorname{BMO}(X)$.

Necessary condition: By Corollary 2.13, (2.2) and Lemma 2.9, we have

$$
\begin{aligned}
& \mu\left(\left\{x \in X: C_{b}(f)(x)>\lambda\right\}\right) \\
& \leq \mu\left(\left\{x \in X: M^{2} f(x)>\frac{\lambda}{C\|b\|_{\mathrm{BMO}(X)}}\right\}\right) \\
& \leq \mu\left(\left\{x \in X: M_{L \log L} f(x)>\frac{\lambda}{C\|b\|_{\mathrm{BMO}(X)}}\right\}\right) \\
& \leq C \int_{X} \frac{C\|b\|_{\mathrm{BMO}(X)}|f(y)|}{\lambda}\left(1+\log ^{+}\left(\frac{C\|b\|_{\mathrm{BMO}(X)}|f(y)|}{\lambda}\right)\right) d \mu(y) .
\end{aligned}
$$

Since for any $\alpha, \beta>0$,

$$
1+\log ^{+}(\alpha \beta) \leq\left(1+\log ^{+} \alpha\right)\left(1+\log ^{+} \beta\right)
$$

we have

$$
\begin{aligned}
& \mu\left(\left\{x \in X: C_{b}(f)(x)>\lambda\right\}\right) \\
& \leq C\|b\|_{\mathrm{BMO}(X)}\left(1+\log ^{+}\|b\|_{\mathrm{BMO}(X)}\right) \int_{X} \frac{|f(y)|}{\lambda}\left(1+\log ^{+}\left(\frac{|f(y)|}{\lambda}\right)\right) d \mu(y) .
\end{aligned}
$$

This finishes the proof of Theorem 2.5.

Proof of Theorem 2.3. By Lemma 2.11, we have

$$
\begin{aligned}
& \mu(\{x \in X:|[M, b] f(x)|>\lambda\}) \\
& \leq \mu\left(\left\{x \in X: C_{b}(f)(x)>\frac{\lambda}{2}\right\}\right)+\mu\left(\left\{x \in X: 2 b^{-}(x) M f(x)>\frac{\lambda}{2}\right\}\right) \\
& \leq \mu\left(\left\{x \in X: C_{b}(f)(x)>\frac{\lambda}{2}\right\}\right)+\mu\left(\left\{x \in X: 2\left\|b^{-}\right\|_{L^{\infty}(X)} M f(x)>\frac{\lambda}{2}\right\}\right) .
\end{aligned}
$$

By (2.5) and (2.6), we can see that

$$
\begin{aligned}
& \mu\left(\left\{x \in X: C_{b}(f)(x)>\frac{\lambda}{2}\right\}\right) \\
& \leq C\|b\|_{\mathrm{BMO}(X)}\left(1+\log ^{+}\|b\|_{\mathrm{BMO}(X)}\right) \int_{X} \frac{|f(y)|}{\lambda}\left(1+\log ^{+}\left(\frac{|f(y)|}{\lambda}\right)\right) d \mu(y) .
\end{aligned}
$$

Since $M$ is weak-type $(1,1)$, we have

$$
\mu\left(\left\{x \in X: 2\left\|b^{-}\right\|_{L^{\infty}(X)} M f(x)>\frac{\lambda}{2}\right\}\right) \leq C\left\|b^{-}\right\|_{L^{\infty}(X)} \int_{X} \frac{|f(y)|}{\lambda} d \mu(y) .
$$

Therefore,

$$
\mu(\{x \in X:|[M, b] f(x)|>\lambda\})
$$


$\leq C\left(\left\|b^{+}\right\|_{\mathrm{BMO}(X)}+\left\|b^{-}\right\|_{L^{\infty}}\right)\left(1+\log ^{+}\left\|b^{+}\right\|_{\mathrm{BMO}(X)}\right) \int_{X} \frac{|f(x)|}{\lambda}\left(1+\log ^{+}\left(\frac{|f(x)|}{\lambda}\right)\right) d x$.

The proof of Theorem 2.3 is complete.

Remark 2.17. We now show that in the general setting of space of homogeneous type, $[M, b]$ fails to be of weak type $(1,1)$. We provide a counter example as follows. Assume that $(X, \rho, \mu)$ is a space of homogeneous type, where $\mu$ satisfies that, for any $x_{0} \in X, \frac{\log t}{\mu\left(B\left(x_{0}, t\right)\right)}$ is decreasing on $(1, \infty)$. Let $b(x)=\log \left(1+d\left(x, x_{0}\right)\right) \in \operatorname{BMO}(X)$ and let $f(x)=\chi_{B\left(x_{0}, 1\right)}(x)$. Then for any $x \notin B\left(x_{0}, 2\right)$,

$$
M f(x)=\sup _{B \ni x} \frac{1}{\mu(B)} \int_{B \cap B\left(x_{0}, 1\right)} d \mu(y)=\sup _{B \ni x} \frac{\mu\left(B \cap B\left(x_{0}, 1\right)\right)}{\mu(B)} .
$$

So

$$
b(x) M(f)(x) \geq \log \left(1+d\left(x, x_{0}\right)\right) \sup _{B \ni x} \frac{\mu\left(B \cap B\left(x_{0}, 1\right)\right)}{\mu(B)} .
$$

On the other hand, for any $x \notin B\left(x_{0}, 2\right)$,

$$
M(b f)(x)=\sup _{B \ni x} \frac{1}{\mu(B)} \int_{B \cap B\left(x_{0}, 1\right)} \log \left(1+d\left(y, x_{0}\right)\right) d \mu(y) \leq \log 2 \sup _{B \ni x} \frac{\mu\left(B \cap B\left(x_{0}, 1\right)\right)}{\mu(B)} .
$$

Therefore, for any $x \notin B\left(x_{0}, 2\right)$,

$$
|[M, b] f(x)|=|M(b f)(x)-b(x) M f(x)| \geq\left|\log \left(1+d\left(x, x_{0}\right)\right)-\log 2\right| \sup _{B \ni x} \frac{\mu\left(B \cap B\left(x_{0}, 1\right)\right)}{\mu(B)} .
$$

Next, it is clear that for $x \notin B\left(x_{0}, 2\right)$,

$$
C_{0} \frac{\mu\left(B\left(x_{0}, 1\right)\right)}{\mu\left(x_{0}, d\left(x, x_{0}\right)\right)} \leq \frac{\mu\left(B\left(x_{0}, 1\right)\right)}{\mu\left(B_{x}\right)} \leq \sup _{B \ni x} \frac{\mu\left(B \cap B\left(x_{0}, 1\right)\right)}{\mu(B)} \leq 1,
$$

where $B_{x}$ is the ball containing $x$ and $B\left(x_{0}, 1\right)$. Hence, we see that for any $x \notin B\left(x_{0}, 100\right)$,

$$
|[M, b] f(x)| \geq \frac{C_{0}}{2} \log \left(1+d\left(x, x_{0}\right)\right) \frac{\mu\left(B\left(x_{0}, 1\right)\right)}{\mu\left(B\left(x_{0}, d\left(x, x_{0}\right)\right)\right)} \geq C_{1} \frac{\log \left(d\left(x, x_{0}\right)\right) \mu\left(B\left(x_{0}, 1\right)\right)}{\mu\left(B\left(x_{0}, d\left(x, x_{0}\right)\right)\right)} .
$$

Therefore, for any $\lambda>0$,

$$
\begin{aligned}
& \lambda \mu(\{x \in X:|[M, b] f(x)|>\lambda\}) \\
& \geq \lambda \mu\left(\left\{x \in X \backslash B\left(x_{0}, 100\right):|[M, b] f(x)|>\lambda\right\}\right) \\
& \geq \lambda \mu\left(\left\{x \in X \backslash B\left(x_{0}, 100\right): C_{1} \frac{\log \left(d\left(x, x_{0}\right)\right) \mu\left(B\left(x_{0}, 1\right)\right)}{\mu\left(B\left(x_{0}, d\left(x, x_{0}\right)\right)\right)}>\lambda\right\}\right) \\
& =\lambda \mu\left(\left\{x \in X \backslash B\left(x_{0}, 100\right): C_{2} \frac{\log \left(d\left(x, x_{0}\right)\right)}{\mu\left(B\left(x_{0}, d\left(x, x_{0}\right)\right)\right)}>\lambda\right\}\right) .
\end{aligned}
$$

Let $\varphi(t)=\frac{\log t}{\mu\left(B\left(x_{0}, t\right)\right)}$. Then it is a decreasing function on $(100, \infty)$, and

$$
\begin{aligned}
& \lambda \mu(\{x \in X:|[M, b] f(x)|>\lambda\}) \\
& \geq \lambda \mu\left(B\left(x_{0}, \varphi^{-1}\left(\frac{\lambda}{C_{2}}\right)\right)\right)-\lambda \mu\left(B\left(x_{0}, 100\right)\right) .
\end{aligned}
$$

Therefore,

$$
\lim _{\lambda \rightarrow 0} \lambda \mu\left(B\left(x_{0}, \varphi^{-1}\left(\frac{\lambda}{C_{2}}\right)\right)\right)=\lim _{t \rightarrow \infty} C_{2} \mu\left(B\left(x_{0}, t\right)\right) \varphi(t)=\lim _{t \rightarrow \infty} \log t=\infty .
$$


We provide a natural example of space of homogeneous type $(X, \rho, \mu)$ beyond the Euclidean setting, such that $\mu$ satisfies the assumption as in the remark above: for any $x_{0} \in X$, $\frac{\log t}{\mu\left(B\left(x_{0}, t\right)\right)}$ is decreasing on $(1, \infty)$.

Example 2.18. We recall the Bessel operator and its underlying space studied by Muckenhoupt and Stein $\left([34)\right.$.Consider $\mathbb{R}_{+}=(0, \infty)$. For $\lambda>-\frac{1}{2}$, the Bessel operator $\Delta_{\lambda}$ on $\mathbb{R}_{+}$ is defined by

$$
\Delta_{\lambda}=-\frac{d^{2}}{d x^{2}}-\frac{2 \lambda}{x} \frac{d}{d x} .
$$

It is a formally self-adjoint operator in $L^{2}\left(\mathbb{R}_{+}, d m_{\lambda}\right)$, where $d m_{\lambda}(x)=x^{2 \lambda} d x$. It is clear that the corresponding underlying space $\left(\mathbb{R}_{+},|\cdot|, d m_{\lambda}\right)$ is a space of homogeneous type in the sense of Coifman and Weiss.

For any $x \in \mathbb{R}_{+}$and $r>0$, let $I(x, r)=(x-r, x+r) \cap \mathbb{R}_{+}$. When $r \leq x$, by mean value theorem, we have

$$
m_{\lambda}(I(x, r))=\int_{x-r}^{x+r} y^{2 \lambda} d y=\frac{1}{2 \lambda+1}\left[(x+r)^{2 \lambda+1}-(x-r)^{2 \lambda+1}\right]=2(x+\theta r)^{2 \lambda} r,
$$

for some $0<\theta<1$. When $r>x$,

$$
m_{\lambda}(I(x, r))=\int_{0}^{x+r} y^{2 \lambda} d y=\frac{1}{2 \lambda+1}(x+r)^{2 \lambda+1} .
$$

Therefore, we can see that $\frac{\log r}{m_{\lambda}(I(x, r))}$ is decreasing on $(1, \infty)$.

\section{Weighted VERSion OF THE COMMUTATOR THEOREMS}

We will further provide the weighted version of Theorems 2.1, 2.2 and 2.4 by establishing new characterisations of $\mathrm{BMO}(X)$ via having the Muckenhoupt weight in both the denominator and the integrand in the definition of BMO norm.

We recall that a locally integrable function $w: X \rightarrow(0,+\infty)$ is an $\mathcal{A}_{p}$ weight (or $w \in \mathcal{A}_{p}$ ), $1<p<\infty$ if

$$
[w]_{\mathcal{A}_{p}}:=\sup _{B}\left(\frac{1}{\mu(B)} \int_{B} w(x) d \mu(x)\right)\left(\frac{1}{\mu(B)} \int_{B} w(x)^{-1 /(p-1)} d \mu(x)\right)^{p-1}<\infty .
$$

A function $w(x) \geq 0$ is called an $\mathcal{A}_{1}$ weight (or $w \in \mathcal{A}_{1}$ ) if $M(w)(x) \leq C w(x)$ for $x \in X$. The class of $\mathcal{A}_{p}$ weights is increasing with $p$ for $1 \leq p<\infty$. The next lemma is a weighted version of Lemma 2.15.

Lemma 3.1. Let $b \in \mathrm{BMO}(X)$ be a nonnegative function, $w$ be an $\mathcal{A}_{q}$ weight and $1<q<$ $\infty$. Suppose that $T$ is a quasilinear operator satisfying (a)-(c) from Section $\mathbb{Q}$ and is bounded on $L^{q}(X, w d \mu)$. Then $[T, b]$ is bounded on $L^{q}(X, w d \mu)$.

Proof. We use a similar argument to [35, Theorem 4.4]. Let $d$ be a real constant and $m(x)=e^{d b(x)}$ for $x \in X$. We claim that if $|d| \leq C_{3} / \delta^{\prime}$ then $m w \in \mathcal{A}_{q}$. Let $\delta>1$ be such that $w^{\delta} \in \mathcal{A}_{q}, \alpha_{1}(B)=\frac{1}{\mu(B)} \int_{B} w(x) e^{d b(x)} d \mu(x)$ and

$$
\alpha_{2}(B)=\left(\frac{1}{\mu(B)} \int_{B} w(x)^{-1 /(q-1)} e^{-d b(x) /(q-1)} d \mu(x)\right)^{q-1},
$$

for any ball $B \subset X$. Using Holder's inequality with $\delta>1$ and its conjugate $\delta^{\prime}\left(\frac{1}{\delta}+\frac{1}{\delta^{\prime}}=1\right)$ in $\alpha_{1}(B)$ and $\alpha_{2}(B)$, we get for any ball $B \subset X$, 


$$
\alpha_{1}(B) \leq\left(\frac{1}{\mu(B)} \int_{B} w(x)^{\delta} d \mu(x)\right)^{1 / \delta}\left(\frac{1}{\mu(B)} \int_{B} e^{d b(x) \delta^{\prime}} d \mu(x)\right)^{1 / \delta^{\prime}}
$$

and

$$
\alpha_{2}(B) \leq\left(\frac{1}{\mu(B)} \int_{B} w(x)^{-\delta /(q-1)} d \mu(x)\right)^{(q-1) / \delta}\left(\frac{1}{\mu(B)} \int_{B} e^{-d b(x) \delta^{\prime} /(q-1)} d \mu(x)\right)^{(q-1) / \delta^{\prime}} .
$$

Let $m_{B}(b)=\frac{1}{\mu(B)} \int_{B} b(x) d \mu(x)$. Observe that

$$
\begin{aligned}
& \left(\frac{1}{\mu(B)} \int_{B} e^{d b(x) \delta^{\prime}} d \mu(x)\right)\left(\frac{1}{\mu(B)} \int_{B} e^{-d b(x) \delta^{\prime} /(q-1)} d \mu(x)\right)^{(q-1)} \\
& \leq \frac{1}{\mu(B)} \int_{B} e^{d\left(b(x)-m_{B}(b)\right) \delta^{\prime}} d \mu(x)\left(\frac{1}{\mu(B)} \int_{B} e^{-d\left(b(x)-m_{B}(b)\right) \delta^{\prime} /(q-1)} d \mu(x)\right)^{(q-1)} \\
& \leq \frac{1}{\mu(B)} \int_{B} e^{|d| \cdot\left|b(x)-m_{B}(b)\right| \delta^{\prime}} d \mu(x)\left(\frac{1}{\mu(B)} \int_{B} e^{|d| \cdot\left|b(x)-m_{B}(b)\right| \delta^{\prime} /(q-1)} d \mu(x)\right)^{(q-1)} .
\end{aligned}
$$

Using Lemma 2.7, we obtain that

$$
\sup _{B} \frac{1}{\mu(B)} \int_{B} e^{|d| \cdot\left|b(x)-m_{B}(b)\right| \delta^{\prime}} d \mu(x) \leq C_{4},
$$

for $|d| \leq C_{3} / \delta^{\prime}$. If $q \geq 2$ then,

$$
\begin{aligned}
\sup _{B}\left(\frac{1}{\mu(B)} \int_{B} e^{|d| \cdot\left|b(x)-m_{B}(b)\right| \delta^{\prime} /(q-1)} d \mu(x)\right)^{(q-1)} & \leq \sup _{B}\left(\frac{1}{\mu(B)} \int_{B} e^{|d| \cdot\left|b(x)-m_{B}(b)\right| \delta^{\prime}} d \mu(x)\right) \\
& \leq C_{4} .
\end{aligned}
$$

The same inequality holds for $1<q<2$ with $|d| \leq C_{3} / \delta^{\prime}$, since for $q^{\prime}=\frac{q}{q-1}$ the conjugate of $q$, we have

$$
\left[e^{|d| \cdot\left|b(x)-m_{B}(b)\right| \delta^{\prime}}\right]_{\mathcal{A}_{q}}=\left[e^{-|d| \cdot\left|b(x)-m_{B}(b)\right| \delta^{\prime} /\left(q^{\prime}-1\right)}\right]_{\mathcal{A}_{q^{\prime}}}^{q^{\prime}-1} .
$$

Now, as $w^{\delta} \in \mathcal{A}_{q}$, we obtain that

$$
\sup _{B}\left(\frac{1}{\mu(B)} \int_{B} w(x)^{\delta} d \mu(x)\right)^{1 / \delta}\left(\frac{1}{\mu(B)} \int_{B} w(x)^{-\delta /(q-1)}\right)^{(q-1) / \delta}<\infty .
$$

It follows that

$$
\sup _{B} \alpha_{1}(B) \alpha_{2}(B) \leq C_{5}
$$

Choose $d$ with $|d| \leq C_{3} / \delta^{\prime}$. We apply [35, Theorem 4.4] with the pair of Banach spaces $\bar{A}=\left(L^{p}\left(X, e^{-d b} \omega d \mu\right), L^{p}\left(X, e^{d b} \omega d \mu\right)\right)$ using that $\bar{A}_{1 / 2, q}=L^{q}(X, w d x)$ to obtain that $[T, b]$ is bounded on $L^{q}(X, w d x)$. The proof of Lemma 3.1 is complete.

The same result holds if $b \in \operatorname{BMO}(X)$ with $b^{-} \in L^{\infty}(X)$ using the inequality

$$
|[T, b] f-[T,|b|] f| \leq 2\left(b^{-} T(f)+T\left(b^{-} f\right)\right)
$$

for $f \in L^{p}(X, w d \mu)$. 
Theorem 3.2. Let $b$ be a real valued, locally integrable function in $X$ and $w$ an $\mathcal{A}_{p}$ weight for $1<p<\infty$. The following assertions are equivalent:

(i) $b \in \operatorname{BMO}(X)$ and $b^{-} \in L^{\infty}(X)$;

(ii) The commutator $\left[M_{p}, b\right]$ is bounded on $L^{q}(X, w d \mu)$, for all $q, p<q<\infty$;

(iii) The commutator $\left[M_{p}, b\right]$ is bounded on $L^{q}(X, w d \mu)$, for some $q, p<q<\infty$.

(iv) For all $q \in[1, \infty)$, we have

$$
\sup _{B} \frac{1}{w(B)} \int_{B}\left|b(x)-M_{p}\left(b \chi_{B}\right)(x)\right|^{q} w(x) d \mu(x)<\infty ;
$$

(v) There exists $q \in[1, \infty)$ such that

$$
\sup _{B} \frac{1}{w(B)} \int_{B}\left|b(x)-M_{p}\left(b \chi_{B}\right)(x)\right|^{q} w(x) d \mu(x)<\infty .
$$

Proof of Theorem 3.2. It is clear that (ii) implies (iii). For (i) $\Rightarrow\left(\right.$ ii), since $M_{p}$ satisfies (a)(c) and is bounded on $L^{q}(X, w d \mu)$ for $p<q<\infty$, the result follows from Lemma 3.1. For $($ iii $) \Rightarrow(\mathrm{v})$, as in the unweighted case, one can write $\left(b \chi_{B}-M_{p, B}(b)\right) \chi_{B}=\left(b M_{p}\left(\chi_{B}\right)-\right.$ $\left.M_{p}\left(b \chi_{B}\right)\right) \chi_{B}=\left[M_{p}, b\right]\left(\chi_{B}\right) \chi_{B}$ and

$$
\begin{aligned}
\int_{B}\left|b-M_{p, B}(b)\right|{ }^{q} w d \mu & =\int_{B}\left|\left[M_{p}, b\right]\left(\chi_{B}\right)\right|^{q} w d \mu \\
& \leq\left\|\left[M_{p}, b\right]\left(\chi_{B}\right)\right\|_{L^{q}(X, w d \mu)}^{q} \leq C\left\|\chi_{B}\right\|_{L^{q}(X, w d \mu)}^{q},
\end{aligned}
$$

where the last inequality follows from the boundedness of $\left[M_{p}, b\right]$ on $L^{q}(X, w d \mu)$. As $\left\|\chi_{B}\right\|_{L^{q}(X, w d \mu)}^{q}=\nu(B)$, we obtain (v). For $(\mathrm{v}) \Rightarrow(\mathrm{i})$, let $B$ be a fixed ball. By Hölder's inequality, we have

$$
\begin{aligned}
\frac{1}{\mu(B)} \int_{B}\left|b-M_{p, B}(b)\right| d \mu(x) & =\frac{1}{\mu(B)} \int_{B}\left|b-M_{p, B}(b)\right| w^{1 / q}(x) w(x)^{-1 / q} d \mu(x) \\
& \leq\left(\frac{1}{\mu(B)} \int_{B}\left|b-M_{p, B}(b)\right|^{q} w(x) d \mu(x)\right)^{\frac{1}{q}} \\
& \times\left(\frac{1}{\mu(B)} \int_{B} w(x)^{-q^{\prime} / q} d \mu(x)\right)^{1 / q^{\prime}} \\
& \leq K\left(\frac{1}{\mu(B)} \int_{B}\left|b-M_{p, B}(b)\right|^{q} w(x) d \mu(x)\right)^{\frac{1}{q}} \\
& \times\left(\frac{1}{\mu(B)} \int_{B} w(x) d \mu(x)\right)^{-1 / q} \\
& \leq K\left(\frac{1}{w(B)} \int_{B}\left|b-M_{p, B}(b)\right|^{q} w(x) d \mu(x)\right)^{\frac{1}{q}} \\
& \leq C .
\end{aligned}
$$

Now, in the same way as the unweighted version, we have

$$
\begin{aligned}
\frac{1}{\mu(B)} \int_{B}\left|b-m_{B}(b)\right| d \mu & =\int_{E_{1}}\left|b-M_{p, B}(b)\right| d \mu \\
& \leq \frac{2}{\mu(B)} \int_{B}\left|b-M_{p, B}(b)\right| d \mu \leq C .
\end{aligned}
$$

Therefore, $b \in \operatorname{BMO}(X)$. In the same way as the unweighted case, $b^{-} \in L^{\infty}(X)$. The implication of (ii) $\Rightarrow($ iv) is similar to (iii) $\Rightarrow(v)$. This completes the proof of Theorem 3.2 . 
Theorem 3.3. Let $b$ be a real valued, locally integrable function in $X$ and $w$ be an $\mathcal{A}_{1}$ weight. The following assertions are equivalent:

(i) $b \in \operatorname{BMO}(X)$ and $b^{-} \in L^{\infty}(X)$;

(ii) The commutator $\left[M^{\sharp}, b\right]$ is bounded on $L^{q}(X, w d \mu)$, for all $q, 1<q<\infty$;

(iii) The commutator $\left[M^{\sharp}, b\right]$ is bounded on $L^{q}(X, w d \mu)$, for some $q, 1<q<\infty$;

(iv) For all $q \in[1, \infty)$, we have

$$
\sup _{B} \frac{1}{w(B)} \int_{B}\left|b(x)-2 M^{\sharp}\left(b \chi_{B}\right)(x)\right|^{q} w(x) d \mu(x)<\infty ;
$$

(v) There exists $q \in[1, \infty)$ such that

$$
\sup _{B} \frac{1}{w(B)} \int_{B}\left|b(x)-2 M^{\sharp}\left(b \chi_{B}\right)(x)\right|^{q} w(x) d \mu(x)<\infty .
$$

Proof of Theorem 3.3. Obviously, (ii) $\Rightarrow$ (iii), and (iv) $\Rightarrow$ (v).

For (i) $\Rightarrow\left(\right.$ ii), since $M^{\sharp}$ satisfies (a)-(c) and is bounded on $L^{q}(X, w d \mu)$, the result follows from Lemma 3.1 .

(iii) $\Rightarrow(\mathrm{v})$. Let $B$ be a fixed ball. We know from the Proof of Theorem 2.2 that $M^{\sharp}\left(\chi_{B}\right)(x)=$ $\frac{1}{2}$, for all $x \in B$ and $\frac{1}{2} b \chi_{B}-M^{\sharp}\left(b \chi_{B}\right) \chi_{B}=\left[M^{\sharp}, b\right]\left(\chi_{B}\right) \cdot \chi_{B}$. By the assumption of (iii), one has

$$
\begin{aligned}
& \left(\int_{B}\left|\frac{1}{2} b(x)-M^{\sharp}\left(b \chi_{B}\right)(x)\right|^{q} w(x) d \mu(x)\right)^{1 / q} \\
& =\left\|\left[M^{\sharp}, b\right]\left(\chi_{B}\right) \chi_{B}\right\|_{L^{q}(X, w d \mu)} \leq\left\|\left[M^{\sharp}, b\right]\left(\chi_{B}\right)\right\|_{L^{q}(X, w d \mu)} \\
& \leq C\left\|\chi_{B}\right\|_{L^{q}(X, w d \mu)} \\
& \leq C w(B)^{1 / q},
\end{aligned}
$$

which implies (iv). For $(\mathrm{v}) \Rightarrow(\mathrm{i})$, from the Proof of Theorem 2.2, we have

$$
\left|m_{B}(b)\right| \leq 2 M^{\sharp}\left(b \chi_{B}\right)(x), \quad x \in B .
$$

To show that $b \in \operatorname{BMO}(X)$, we use the same arguments as in the Proof of Theorem 2.2 and Theorem 3.2 with the set $E=\left\{x \in B: b(x) \leq m_{B}(b)\right\}$. For $\frac{1}{q}+\frac{1}{q^{\prime}}=1$, we get

$$
\begin{aligned}
& \frac{1}{\mu(B)} \int_{B}\left|b(x)-m_{B}(b)\right| d \mu(x) \\
& =\frac{2}{\mu(B)} \int_{E}\left(b(x)-m_{B}(b)\right) d \mu(x) \\
& \leq \frac{2}{\mu(B)} \int_{E}\left(2 M^{\sharp}\left(b \chi_{B}\right)(x)-b(x)\right) d \mu(x) \\
& \leq \frac{2}{\mu(B)} \int_{B}\left|2 M^{\sharp}\left(b \chi_{B}\right)(x)-b(x)\right| w(x)^{1 / q} w(x)^{-1 / q} d \mu(x) \\
& \leq 2\left(\frac{1}{\mu(B)} \int_{B}\left|2 M^{\sharp}\left(b \chi_{B}\right)(x)-b(x)\right|^{q} w(x) d \mu(x)\right)^{1 / q}\left(\frac{1}{\mu(B)} \int_{B} w(x)^{-q^{\prime} / q} d \mu(x)\right)^{1 / q^{\prime}} \\
& \leq 2\left(\frac{1}{\mu(B)} \int_{B}\left|2 M^{\sharp}\left(b \chi_{B}\right)(x)-b(x)\right|^{q} w(x) d \mu(x)\right)^{1 / q} \frac{[w]_{\mathcal{A}_{q}}^{1 / q}}{\mu(B)^{-1 / q}}\left(\int_{B} w(x) d \mu(x)\right)^{-1 / q} \\
& \leq K\left(\frac{1}{w(B)} \int_{B}\left|2 M^{\sharp}\left(b \chi_{B}\right)(x)-b(x)\right|^{q} w(x) d \mu(x)\right)^{1 / q} \\
& \leq C .
\end{aligned}
$$


One can prove in the same way as in the Proof of Theorem 2.2 that $b^{-} \in L^{\infty}(X)$. The implication of (ii) $\Rightarrow$ (iv) is similar to (iii) $\Rightarrow(v)$, which ends the proof of Theorem 3.3 ,

Theorem 3.4. Let $b \in L_{\text {loc }}^{1}(X), 1<p<\infty$ and $w \in \mathcal{A}_{p}$. Then the maximal commutator $C_{b}$ is bounded on $L^{p}(X, w d \mu)$ if and only if $b \in \mathrm{BMO}(\mathrm{X})$.

Proof of Theorem 3.4. Sufficient condition: It follows the same argument as in the unweighted case. If $b \in \mathrm{BMO}(X)$, by Corollary 2.13 and the boundedness of $M$ on $L^{p}(X, w d \mu)$, we obtain that, for every $f \in L^{p}(X, w d \mu), 1<p<\infty$,

$$
\left\|C_{b}(f)\right\|_{L^{p}(X, w d \mu)} \leq C\|b\|_{\mathrm{BMO}(X)}\left\|M^{2} f\right\|_{L^{p}(X, w d \mu)} \leq C\|b\|_{\mathrm{BMO}(X)}\|f\|_{L^{p}(X, w d \mu)} .
$$

Necessary condition: Assume that $C_{b}$ is bounded on $L^{p}(X, w d \mu)$. By Hölder's inequality, we get

$$
\begin{aligned}
& \inf _{c \in \mathbb{R}} \int_{B}|b(x)-c| d \mu(x) \\
& \leq \inf _{y \in B} \int_{B}|b(x)-b(y)| d \mu(x) \\
& \leq \frac{1}{\mu(B)} \int_{B} \int_{B}|b(x)-b(y)| d \mu(x) d \mu(y) \\
& \leq[w]_{\mathcal{A}_{p}}{ }^{1 / p} w(B)^{-\frac{1}{p}} \mu(B)\left[\int_{B}\left(\frac{1}{\mu(B)} \int_{B}|b(x)-b(y)| d \mu(y)\right)^{p} w(x) d \mu(x)\right]^{\frac{1}{p}} .
\end{aligned}
$$

We use that for any $x \in B, \frac{1}{\mu(B)} \int_{B}|b(x)-b(y)| d \mu(y) \leq C_{b}\left(\chi_{B}\right)(x)$ to obtain that

$$
\begin{aligned}
\frac{1}{\mu(B)} \inf _{c \in \mathbb{R}} \int_{B}|b(x)-c| d \mu(x) & \leq w(B)^{-\frac{1}{p}}\left(\int_{B}\left|C_{b}\left(\chi_{B}\right)(x)\right|^{p} w(x) d \mu(x)\right)^{\frac{1}{p}} \\
& \leq[w]_{\mathcal{A}_{p}}{ }^{1 / p} w(B)^{-\frac{1}{p}}\left\|C_{b}\right\|_{L^{p}(X, w d \mu) \rightarrow L^{p}(X, w d \mu)}\left\|\chi_{B}\right\|_{L^{p}(X, w d \mu)} \\
& \leq[w]_{\mathcal{A}_{p}}{ }^{1 / p}\left\|C_{b}\right\|_{L^{p}(X, w d \mu) \rightarrow L^{p}(X, w d \mu)}
\end{aligned}
$$

Therefore, $b \in \operatorname{BMO}(X)$, and

$$
\|b\|_{\mathrm{BMO}(X)} \leq C\left\|C_{b}\right\|_{L^{p}(X, w d \mu) \rightarrow L^{p}(X, w d \mu)} .
$$

This completes the proof of the theorem.

\section{LOCAL VERSION OF THE COMMUTATOR THEOREMS}

We will sketch the result in the case that $\mu(X)<\infty$ in this section. Note that in this setting, the BMO space $\operatorname{BMO}(X)$ is defined as the set of all $b \in L^{1}(X)$ such that

$$
\sup _{x \in X, r>0, B(x, r) \subset X} \frac{1}{\mu(B(x, r))} \int_{B(x, r)}\left|b(y)-m_{B}(b)\right| d \mu(y)<\infty,
$$

and the norm is defined as

$$
\|b\|_{\mathrm{BMO}(X)}:=\sup _{x \in X, r>0, B(x, r) \subset X} \frac{1}{\mu(B(x, r))} \int_{B(x, r)}\left|b(y)-m_{B}(b)\right| d \mu(y)+\|b\|_{L^{1}(X)} .
$$

Theorem 4.1. Suppose $\mu(X)<\infty$, $\operatorname{diam}(X)<\infty$ and $\operatorname{BMO}(X)$ is defined as above. Then the results in Theorems 2.1, 2.2, 2.3, 2.4 and 2.5 hold in this setting. 
Proof of Theorem 4.1. The proof is similar to those of Theorems 2.1, 2.2, 2.3, 2.4 and 2.5, except the case when proving $b \in \operatorname{BMO}(X)$ in these theorems. By assumption, there exists $R_{0}>0$ such that for any $B(x, r) \subset X$, we have $r<R_{0}$. We test the $\operatorname{BMO}(X)$ condition on the case of balls with big radius and small radius. In the case of balls with small radius, $r<R_{0}$, the proof is the same as in Theorems 2.1, 2.2, 2.3, 2.4 and 2.5. In the case of balls with large radius, $r \geq R_{0}$. By (1.3), we obtain that

$$
\begin{aligned}
\frac{1}{\mu(B)} \int_{B}\left|b(x)-m_{B}(b)\right| d \mu(x) & \leq 2 C_{\mu}\left(\frac{\operatorname{diam}(X)}{r}\right)^{n} \frac{1}{\mu(X)}\|b\|_{L^{1}(X)} \\
& \leq 2 C_{\mu}\left(\frac{\operatorname{diam}(X)}{R_{0}}\right)^{n} \frac{1}{\mu(X)}\|b\|_{L^{1}(X)} .
\end{aligned}
$$

This finishes the proof of Theorem 4.1.

We remark that a concrete example of the space of homogeneous type $(X, d, \mu)$ with $\mu(X)<\infty$ and $\operatorname{diam}(X)<\infty$ is the boundary of a bounded strictly pseudoconvex domain in $\mathbb{C}^{n}$, see for example the recent works in [14, [30] and [32. To be more precise, we recall the bounded domain $D$ from [32] with defining function $\rho$, which means that $D=\left\{z \in \mathbb{C}^{n}\right.$ : $\rho(z)<0\}$ with $\rho: \mathbb{C}^{n} \rightarrow \mathbb{R}$ and boundary $b D$. Without lost of generality, assume that $\rho$ is strictly plurisubharmonic (see [40, Ch. II Sec. 4]). Let $\mathcal{L}_{0}(w, z)$ be the negative of the Levi polynomial at $w \in b D$, given by

$$
\mathcal{L}_{0}(w, z)=\langle\partial \rho(w), w-z\rangle-\frac{1}{2} \sum_{j, k} \frac{\partial^{2} \rho(w)}{\partial w_{j} \partial w_{k}}\left(w_{j}-z_{j}\right)\left(w_{k}-z_{k}\right),
$$

where $\partial \rho(w)=\left(\frac{\partial \rho}{\partial w_{1}}(w), \cdots, \frac{\partial \rho}{\partial w_{n}}(w)\right)$ and we have used the notation $\langle\eta, \zeta\rangle=\sum_{j=1}^{n} \eta_{j} \zeta_{j}$ for $\eta=\left(\eta_{1}, \cdots, \eta_{n}\right), \zeta=\left(\zeta_{1}, \ldots, \zeta_{n}\right) \in \mathbb{C}^{n}$. The strict plurisubharmonicity of $\rho$ implies that

$$
2 \operatorname{Re} \mathcal{L}_{0}(w, z) \geq-\rho(z)+c|w-z|^{2},
$$

for some $c>0$, whenever $w \in b D$ and $z \in \bar{D}$ is sufficiently close to $w$. Then a modification of $\mathcal{L}_{0}$ is as follows

$$
g_{0}(w, z)=\chi \mathcal{L}_{0}+(1-\chi)|w-z|^{2} .
$$

Here $\chi=\chi(w, z)$ is a $C^{\infty}$-cutoff function with $\chi=0$ when $|w-z| \leq \mu / 2$ and $\chi=1$ if $|w-z| \geq \mu$. Then for $\mu$ chosen sufficiently small (and then kept fixed throughout), we have that $\operatorname{Re} g_{0}(w, z) \geq c\left(-\rho(z)+|w-z|^{2}\right)$ for $z$ in $\bar{D}$ and $w$ in $b D$, with $c$ a positive constant.

Note that the modified Levi polynomial $g_{0}$ has no smoothness beyond continuity in the variable $w$. So in 32, for each $\epsilon>0$ the authors considered a variant $g_{\epsilon}$ defined as follows: let $\left\{\tau_{j k}^{\epsilon}(w)\right\}$ be an $n \times n$-matrix of $C^{1}$ functions such that

Set

$$
\sup _{w \in b D}\left|\frac{\partial^{2} \rho(w)}{\partial w_{j} \partial w_{k}}-\tau_{j k}^{\epsilon}(w)\right| \leq \epsilon, \quad 1 \leq j, k \leq n
$$

$$
\mathcal{L}_{\epsilon}(w, z)=\langle\partial \rho(w), w-z\rangle-\frac{1}{2} \sum_{j, k} \tau_{j k}^{\epsilon}(w)\left(w_{j}-z_{j}\right)\left(w_{k}-z_{k}\right),
$$

and define

$$
g_{\epsilon}(w, z)=\chi \mathcal{L}_{\epsilon}+(1-\chi)|w-z|^{2}, \quad z, w \in \mathbb{C}^{n} .
$$

Now $g_{\epsilon}$ is $C^{1}$ in $w$ and $C^{\infty}$ in $z$. We note that

$$
\left|g_{0}(w, z)-g_{\epsilon}(w, z)\right| \lesssim \epsilon|w-z|^{2} .
$$


We shall always assume that $\epsilon$ is sufficiently small, we then have

$$
\left|g_{\epsilon}(w, z)\right| \approx\left|g_{0}(w, z)\right|
$$

where the equivalence $\approx$ is independent of $\epsilon$. Now on the boundary $b D$, define the function $\mathrm{d}(w, z)=\left|g_{0}(w, z)\right|^{\frac{1}{2}}$. According to [32, Proposition 3], d satisfies that for all $w, z, z^{\prime} \in b D$,

(a) $\mathrm{d}(w, z)=0$ iff $w=z$;

(b) $\mathrm{d}(w, z) \approx \mathrm{d}(z, w)$

(c) $\mathrm{d}(w, z) \lesssim \mathrm{d}\left(w, z^{\prime}\right)+\mathrm{d}\left(z^{\prime}, z\right)$.

Next we recall the Leray-Levi measure $d \lambda$ on $b D$ defined via the $(2 n-1)$-form

$$
\frac{1}{(2 \pi i)^{n}} \partial \rho \wedge(\bar{\partial} \partial \rho)^{n-1}
$$

To be more precise, we have the linear functional

$$
f \mapsto \frac{1}{(2 \pi i)^{n}} \int_{b D} f(w) j^{*}\left(\partial \rho \wedge(\bar{\partial} \partial \rho)^{n-1}\right)(w)=: \int_{b D} f(w) d \lambda(w)
$$

defined for $f \in C(b D)$, and this defines the measure $d \lambda$. Then one also has

$$
d \lambda(w)=\frac{1}{(2 \pi i)^{n}} j^{*}\left(\partial \rho \wedge(\bar{\partial} \partial \rho)^{n-1}\right)(w)=\Lambda(w) d \sigma(w),
$$

where $j^{*}$ denotes the pullback under the inclusion $j: b D \hookrightarrow \mathbb{C}^{n}, d \sigma$ is the induced Lebesgue measure on $b D$ and $\Lambda(w)$ is a continuous function such that $c \leq \Lambda(w) \leq \tilde{c}, w \in b D$, with $c$ and $\tilde{c}$ two positive constants.

Example 4.2. Let $(b D, \mathrm{~d}, \lambda)$ be defined as above. Then it is a specific space of homogeneous type with $\lambda(b D)<\infty$ and $\operatorname{diam}(b D)<\infty$.

We also recollect the boundary balls $B_{r}(w)$ determined via the quasidistance d and their measures, i.e.,

$$
B_{r}(w)=\{z \in b D: \mathrm{d}(w, z)<r\}, \quad \text { where } w \in b D .
$$

According to [32, p. 139],

$$
c_{\lambda}^{-1} r^{2 n} \leq \lambda\left(B_{r}(w)\right) \leq c_{\lambda} r^{2 n}, \quad 0<r \leq 1,
$$

for some $c_{\lambda}>1$.

Acknowledgement: This work was supported by Natural Science Foundation of China (Grant Nos. 11671185, 11701250 and 11771195) and Natural Science Foundation of Shandong Province (Grant Nos. ZR2018LA002 and ZR2019YQ04).

\section{REFERENCES}

1. M. Agcayazi, A. Gogatishvili, K. Koca and R. Mustafayev, A note on maximal commutators and commutators of maximal functions. J. Math. Soc. Japan 67 (2) (2015), 581-593.

2. H. Aimar, A. Bernardis, And B. IAfFei, Multiresolution approximations and unconditional bases on weighted Lebesgue spaces on spaces of homogeneous type, J. Approx. Theory 148 (1) (2007), 12-34.

3. A. M. Alphonse, An end point estimate for maximal commutators, J. Fourier Anal. Appl. 6 (4) (2000), 449-456.

4. P. Auscher And T. Hytönen, Orthonormal bases of regular wavelets in spaces of homogeneous type, Appl. Comput. Harmon. Anal. 34 (2) (2013), 266-296.

5. J. Bastero, M. Milman and F. J. Ruiz, Commutators for the maximal and sharp functions, Proc. Amer. Math. Soc. 128 (2000), 3329-3334. 
6. L. Boutet De Monvel And J. Sjöstrand, Sur la singularité des noyaux de Bergman et de Szegö, Astérisque, 34-35 (1976), 123-164.

7. P. Chen, J. Li, and L.A. WArd, BMO from dyadic BMO via expectations on product spaces of homogeneous type, J. Funct. Anal. 265 (2013), 2420-2451.

8. W. Chen And E. SAWYeR, Endpoint estimates for commutators of singular integrals on spaces of homogeneous type, J. Math. Anal. Appl. 282 (2) (2003), 553-566.

9. R. Coifman, R. Rochberg and G. Weiss, Factorization theorems for Hardy spaces in several variables, Ann. Math. 103 (1976), 611-635.

10. R.R. Coifman And G. Weiss, Analyse harmonique non-commutative sur certains espaces homogènes. Étude de certaines intégrales singulières. (French), Lecture Notes in Mathematics, Vol. 242, SpringerVerlag, Berlin, 1971.

11. R.R. Coifman And G. Weiss, Extensions of Hardy spaces and their use in analysis, Bull. Amer. Math. Soc. 83 (1977), 569-645.

12. G. David, J.-L. Journé, And S. Semmes, Calderón-Zygmund operators, para-accretive functions and interpolation, Rev. Mat. Iberoamericana 1 (4) (1985), 1-56.

13. D.G. Deng And Y. Han, Harmonic analysis on spaces of homogeneous type, with a preface by Yves Meyer, Lecture Notes in Mathematics, Vol. 1966, Springer-Verlag, Berlin, 2009.

14. X. T. Duong, M. Lacey, J. Li, B. D. Wick and Q. Y. Wu, Commutators of Cauchy type integrals for domains in $\mathbb{C}^{n}$ with minimal smoothness, Indiana University Math. J. in press.

15. D. E. Edmunds, V. Kokilashvili and A. Meskhi, Weight inequalities for singular integrals defined on spaces of homogeneous and nonhomogeneous type, Georgian Math. J. 8 (1) (2001), 33-59.

16. C. Fefferman, The Bergman kernel and biholomorphic mappings of pseudoconvex domains. Invent. Math., 26 (1974), 1-65.

17. J. García-Cuerva and J. L. Rubio de Francia, Weighted norm inequalities and related topics, North Holland Math. Studies 116, North Holland, Amsterdam, (1985).

18. J. García-Cuerva, E. Harboure, C. Segovia, J.L. Torrea, Weighted norm inequalities for commutators of strongly singular integrals, Indiana Univ. Math. J. 40 (1991), 1397-1420.

19. L. Grafakos, L. G. LiU And D. C. YAng, Vector-valued singular integrals and maximal functions on spaces of homogeneous type, Math. Scand. 104 (2009), no. 2, 296-310.

20. Y. HAN, Calderón-type reproducing formula and the $T_{b}$ theorem, Rev. Mat. Iberoamericana 10 (1994), 51-91.

21. Y. Han, Plancherel-Pôlya type inequality on spaces of homogeneous type and its applications, Proc. Amer. Math. Soc. 126 (1998), no. 11, 3315-3327.

22. Y. Han, J. Li, AND C.-C. Lin, Criterions of the $L^{2}$ boundedness and sharp endpoint estimates for singular integral operators on product spaces of homogeneous type, Ann. Scuola Norm. Sup. Pisa Cl. Sci. 16 (3) (2016), 845-907.

23. Y. HAN AND E.T. SAWYER, Littlewood-Paley theory on spaces of homogeneous type and the classical function spaces, Mem. Amer. Math. Soc. 530 (110) (1994), vi + 126 pp.

24. J. Hart And R. H. Torres, John-Nirenberg inequalities and weight invariant BMO spaces, J. Geom. Anal. 29 (2) (2019), 1608-1648.

25. Z. HE, Y. HAN, J. Li, L. LiU, D. YANG AND W. YuAn, A complete real-variable theory of Hardy spaces on spaces of homogeneous type, J. Fourier Anal. Appl. in press.

26. KwOK-Pun Ho, Characterizations of BMO spaces by $A_{p}$ weights and p-convexity, Hiroshima Math. J. 41 (2011), 153-165.

27. G. Hu, H. Lin And D. YAng, Commutators of the Hardy-Littlewood maximal operator with BMO symbols on spaces of homogeneous type, Abstr. Appl. Anal. 2008 (2008), Art. ID 237937.

28. G. Hu AND D. YANG Maximal commutators of BMO functions and singular integral operators with non-smooth kernels on spaces of homogeneous type, J. Math. Anal. Appl. 354 (1) (2009), 249-262.

29. T. Hytönen And A. Kairema, Systems of dyadic cubes in a doubling metric space, Colloq. Math. 126 (2012), no. 1, 1-33.

30. S. G. Krantz and S.-Y. Li, Boundedness and compactness of integral operators on spaces of homogeneous type and applications, II, J. Math. Anal. Appl., 258 (2001), 642-657.

31. M. Kronz, Some function spaces on spaces of homogeneous type, Manuscripta Math. 106 (2) (2001), 219-248.

32. L. Lanzani and E. Stein, The Cauchy-Szegö projection for domains in $\mathbb{C}^{n}$ with minimal smoothness, Duke Math. J. 166 (2017), 125-176.

33. A. K. Lerner, On weighted estimates of non-increasing rearrangements, East J. Approx. 4 (1998), 277-290.

34. R.A. Macías and C. Segovia, Lipschitz functions on spaces of homogeneous type, Adv. Math. 33 (1979), 257-270. 
35. M. Milman and T. Schonbek, Second order estimates in interpolation theory and applications, Proc. Amer. Math. Soc. 110 (4) (1990), 961-969.

36. A. Nagel, J.-P. Rosay, E. M. Stein and S. Wainger, Estimates for the Bergman and Szegö kernels in $\mathbb{C}^{2}$, Ann. Math. 129 (2) (1989), 113-149.

37. A. Nagel And E.M. Stein, On the product theory of singular integrals, Rev. Mat. Iberoamericana 20 (2004), 531-561.

38. A. Nagel And E.M. Stein, The $\bar{\partial}_{b}$-complex on decoupled boundaries in $\mathbb{C}^{n}$, Ann. Math. 164 (2) (2006), 649-713.

39. G. Pradolini, O. Salinas, Commutators of singular integrals on spaces of homogeneous type, Czechoslovak Math. J. 57 (1) (2007) 75-93.

40. R. M. RANGE, Holomorphic functions and integral representations in several complex variables, Graduate Texts in Mathematics, 108, Springer-Verlag, New York, 1986.

41. M. M. Rao And Z. D. Ren, Theory of Orlicz Spaces, vol. 146 of Monographs and Textbooks in Pure and Applied Mathematics, Marcel Dekker, New York, 1991.

42. E. Stein, Boundary Behavior of Holomorphic Functions of Several Complex Variables, Princeton University Press, Princeton, 1972.

Zunwei Fu, Department of Mathematics, Linyi University, Shandong, 276005, China

E-mail address: fuzunwei@lyu.edu.cn

Elodie Pozzi, Department of Mathematics and Statistics, Saint Louis University, 220 N. Grand Blvd, 63103 St Louis MO, USA

E-mail address: elodie.pozzi@slu.edu

Qingyan Wu, Department of Mathematics, Linyi University, Shandong, 276005, China

E-mail address: wuqingyan@lyu.edu.cn 